\title{
Perennial favourites
}

\author{
This year the United Nations is unwittingly bringing together seaweed, a pair of nineteenth-century glass sculptors, \\ bovine teeth, photosynthetic efficiency and neurodegenerative diseases. Find out how.
}

T he United Nations annually designates a topic or topics as a particular focus for the year ahead, one of which is usually associated with the Food and Agriculture Organization (FAO); 2020 was the International Year of Plant Health, and 2021 was the International Year of Fruits and Vegetables. It was fairly easy to see the involvement of plants in these - and when, in 12 months' time, 2023 ushers in the International Year of Millets, this will again be something to which Nature Plants can hopefully contribute. It is going to be a little more difficult for 2022, as this is the International Year of Artisanal Fisheries and Aquaculture (https://www.fao.org/ artisanal-fisheries-aquaculture-2022/home/).

At first glance this does not seem to be a very plant-orientated topic. Rice cultivation traditionally involves periods of submersion, but that hardly qualifies as aquaculture. Similarly, large-scale hydroponics does not have the 'artisanal' quality that the FAO intends. There are a few crop plants that are aquatic (most notably, watercress, Nasturtium officinale), but probably the closest fit would be the farming of kelp. Kelp production has been growing by $8 \%$ per year recently, and there is a global annual production of around 900,000 tons. Seaweeds can be used as a salad crop, but more frequently kelp is processed for its high sugar content, including polysaccharides such as agar and carrageenan that are used as gelling agents. Most current research on kelp is ecological in nature, as kelp farming can be regenerative to coastlines (especially when used in conjunction with mussel and other molluscan cultivation).

The United Nations has also designated 2022 the International Year of Glass, which is the inspiration for our cover image this month. Glasses are interesting materials, being neither conventional solids nor conventional liquids. Rather they transition from a hard, brittle state into a viscous and pliable form over a range of temperatures. This glass transition has allowed the production of delicate glass sculptures that are particularly evocative of living structures.

Perhaps the greatest exponents of this art were Leopold Blaschka and his son Rudolf. The two worked in Dresden, Germany, during the second half of the nineteenth century and into the early years of the twentieth. The Blaschka family had already been in the glass business for several generations, including the manufacture of glass eyes, by 1853 . Becalmed on a ship to America that year, Leopold became fascinated by the sea creatures he could observe (such as jellyfish) and subsequently began creating models of them in glass. These were sufficiently accurate that various universities acquired sets as teaching aides.

In 1886, Professor George Lincoln Goodale approached the Blaschkas to make models of plants for the newly established Harvard Botanical Museum. Despite an initial reluctance to switch kingdoms, over the next 50 years Leopold and Rudolf made over 4,000 glass models of 780 plant species from 164 families. They illustrated specific organs and showed the processes of pollination and of diseases. These treasures form the Ware Collection, which can still be seen at the Harvard Museum of Natural History (https://hmnh.harvard.edu/ glass-flowers).

Classical glass is made of principally of silica, and silica glass - or something very like it - is found in the leaves of grasses in the form of abrasive phytoliths. These are thought to protect the plants from grazing herbivores, but the evolution of defence strategies almost inevitably spurs the evolution of more sophisticated forms of attack. In this case, phytoliths drove the development of high-crowned cheek teeth in grazing animals, familiar to anyone who has seen a cow or sheep skull.

Glasses can be formed from a variety of materials, especially disordered polymers - a category that includes some natural proteins - and plants contain protein structures with physical properties similar to those of glasses. One such is the pyrenoid, an organelle found in cyanobacteria and algae. The pyrenoid matrix is made up from a mixture of ribulose-1,5-bisphosphate carboxylase/ oxygenase (RUBISCO) and an intrinsically disordered protein called essential pyrenoid component 1 (EPYC1). EPYC1 weakly tethers RUBISCO molecules together so that the pyrenoid behaves as a phase-separated, liquid-like organelle, reminiscent of oil droplets in water (https://www.nature. com/articles/s41477-020-00811-y). During cell division, pyrenoids 'dissolve' into the surrounding chloroplast, re-forming once division is complete. This flexible aggregation of RUBISCO forms part of a $\mathrm{CO}_{2}$ concentration mechanism to overcome the shortcomings of the poor substrate discrimination of this enzyme between $\mathrm{CO}_{2}$ and oxygen. The engineering of pyrenoid structures into the chloroplasts of $\mathrm{C}_{3}$ plants is actively being explored as a way to increase the photosynthetic efficiency of crop plants.

A similar phase separation of an intrinsically disordered protein is found in some seeds, where it is thought to act as a sensor of hydration state (https://www. nature.com/articles/s41477-021-01083-w). The protein, FLOE1, has similarities to the prion protein aggregates that are seen in neurological disorders such as Creutzfeldt-Jakob disease, scrapie and bovine spongiform encephalopathy. In dry seeds, FLOE1 is dispersed throughout the cytoplasm, but it aggregates into globules when the seeds are rehydrated, affecting their germination.

It would be easy to dismiss the 'International Year of' designations as an annual public relations exercise to justify bringing some unarguably worthy topic to public attention. And yet, even in apparently fallow years, they demonstrate that plants are so fundamental to life on this planet that there is a plant angle to everything.

Published online: 24 January 2022 https://doi.org/10.1038/s41477-021-01093-8 